BCCNT: 02/121/318

\title{
Calculation of Temperature-Dependent Hadronic Correlation Functions of Pseudoscalar and Vector Currents
}

\author{
Bing He, Hu Li, C. M. Shakin * and Qing Sun \\ Brooklyn College of the City University of New York \\ Brooklyn, New York 11210
}

(Dated: December, 2002)

\begin{abstract}
We make use of the Nambu-Jona-Lasinio (NJL) formalism and real-time finite-temperature field theory to calculate hadronic current correlation functions in the deconfined phase of quantum chromodynamics (QCD). Here, we consider both pseudoscalar and vector currents. Since the method used in the lattice analysis to calculate the spectral functions requires assumptions about the likelihood of a particular form for the spectral function, we believe our calculations will be useful to researchers who wish to calculate hadronic current correlation functions at finite temperature using lattice-based methods. Our model makes use of temperature-dependent coupling constants for the NJL model. We present various arguments that such temperature dependence is necessary, if the results of the model are to be consistent with what is known concerning QCD thermodynamics.
\end{abstract}

PACS numbers: 12.39.Fe, 12.38.Aw, 14.65.Bt

*email casbc@cunyvm.cuny.edu 


\section{INTRODUCTION}

In recent years we have seen a great deal of interest in the properties of dense matter, with particular attention given to diquark condensation and color superconductivity [1]. Since it is difficult to study the properties of dense matter in lattice simulations of QCD [2], the Nambu-Jona-Lasinio (NJL) model and closely related instanton-based models have been used in such studies. We have become interested in a possible density dependence of the coupling constants of chiral Lagrangian models, since density dependence of the coupling parameters could affect the conclusions drawn from the studies of dense matter. We have introduced density-dependent coupling constants for the NJL model in earlier works [3, 4], and have presented some arguments that such density dependence may be necessary [4]. However, it is much easier to discuss the temperature dependence of NJL coupling parameters, rather that the density dependence, since a good deal is known concerning finite-temperature QCD thermodynamics [5]. In particular, the study of a gluon gas at high temperature suggests that the system is well described as weakly interacting for $T \gtrsim 6 T_{c}$, where $T_{c}$ is the temperature of the confinement-deconfinement phase transition. (See Fig.1.3 of Ref. [5].)

In this work we suggest that rather straightforward calculations of hadronic current correlation functions at finite temperature can provide information concerning a possible temperature dependence of the NJL coupling parameters. We perform calculations of hadronic current correlation functions for pseudoscalar and vector currents in the range $1.2 \leqslant T / T_{c} \leqslant 5.88$. We make use of two models for the temperature dependence of the NJL coupling parameters. For model 1 , we use $G(T)=G\left[1-0.17\left(T / T_{c}\right)\right]$, which was the form used in our previous studies of meson properties [6] and hadronic current correlation functions [7] at finite temperature. In this work we also introduce a model 2, for which

$G(T)=G\left[1-0.0289\left(T / T_{c}\right)^{2}\right]$. In both cases, $G(T)=0$ for $T / T_{c}=5.88$. For values of $T / T_{c}>5.88$ we put $G(T)=0$ for both models.

For the sake of completeness we present the Lagrangian of a generalized NJL model that 
we have used in our studies of meson properties at finite temperature and density

$$
\begin{aligned}
\mathcal{L}= & \bar{q}\left(i \not \partial-m^{0}\right) q+\frac{G_{S}}{2} \sum_{i=0}^{8}\left[\left(\bar{q} \lambda^{i} q\right)^{2}+\left(\bar{q} i \gamma_{5} \lambda^{i} q\right)^{2}\right] \\
& -\frac{G_{V}}{2} \sum_{i=0}^{8}\left[\left(\bar{q} \lambda^{i} \gamma_{\mu} q\right)^{2}+\left(\bar{q} \lambda^{i} \gamma_{5} \gamma_{\mu} q\right)^{2}\right] \\
& +\frac{G_{D}}{2}\left\{\operatorname{det}\left[\bar{q}\left(1+\gamma_{5}\right) q\right]+\operatorname{det}\left[\bar{q}\left(1-\gamma_{5}\right) q\right]\right\} \\
& +\mathcal{L}_{\text {conf }} .
\end{aligned}
$$

Here, $m^{0}$ is a current quark mass matrix, $m^{0}=\operatorname{diag}\left(m_{u}^{0}, m_{d}^{0}, m_{s}^{0}\right)$. The $\lambda^{i}$ are the GellMann (flavor) matrices. Here, $\lambda^{0}=\sqrt{2 / 3} \mathbf{1}$ with 1 being the unit matrix. The fourth term on the right-hand side of Eq. (1.1) is the 't Hooft interaction. Finally, $\mathcal{L}_{\text {conf }}$ represents the model of confinement we have used in our work.

In order to specify the parameters in Eq. (1.1) we may refer to Ref [8]. There, a quite detailed fit was made to the properties of the $\eta$ mesons. Our analysis yields fits the first four experimentally known levels and provides excellent fits to the mixing angles and decay constants of the $\eta(547)$ and $\eta \prime(958)$. That work led to the specification of $G_{S}=11.84$ $\mathrm{GeV}^{-2}$. The 't Hooft interaction strength, $G_{D}$, was taken to be in the range $-220 \mathrm{GeV}^{-5}$ $\leq G_{D} \leq-180 \mathrm{GeV}^{-5}$ when calculating the properties of the $\eta$ mesons. We also used $G_{V}=13.0 \mathrm{GeV}^{-2}$ in Ref.[8]. (It is worth noting that Hatsuda and Kunihiro [9] have used a sharp moment cutoff of $\Lambda_{3}=0.6314 \mathrm{GeV}$ and a value of $G_{D}=185.1 \mathrm{GeV}^{-5}$. Their value of $G_{S}$ is smaller than ours, since they do not include a model of confinement in their analysis.)

In terms of these parameters we can introduce effective coupling constants for states with particular quantum numbers. The effective coupling parameter involves the vacuum condensates $\alpha=<0|\bar{u} u| 0>, \beta=<0|\bar{d} d| 0>$ and $\gamma=<0|\bar{s} s| 0>$. For the pseudoscalarisovector states, we have [9]

$$
G_{P}^{e f f}=G_{S}+\gamma \frac{G_{D}}{2}
$$

We take $\alpha=\beta=-(0.241 \mathrm{GeV})^{3}$ and $\gamma=-(0.258 \mathrm{GeV})^{3}[10]$. If we put $G_{D}=-190 \mathrm{GeV}^{-5}$, we find $G_{P}=13.47 \mathrm{GeV}^{-2}$.

In Ref. [6] we presented relativistic random phase approximation (RPA) calculations for the pion, kaon and several other mesons. It was found that in the case of pion, that the use of $G_{P}^{e f f}=13.49 \mathrm{GeV}^{-2}$ gave a pion energy of $140 \mathrm{MeV}$ at $T=0$. It is gratifying to see that 
an independent calculation gives rise to essentially the same effective coupling constant as that obtained from the parameters determined in Ref.[8].

If we consider the scalar-isovector states, the effective coupling constant is

$$
G_{S}^{e f f}=G_{S}-\gamma \frac{G_{D}}{2}
$$

which, for $G_{S}=-11.84 \mathrm{GeV}^{-2}, G_{D}=190.0 \mathrm{GeV}^{-5}$ and $\gamma=-(0.258 \mathrm{GeV})^{3}$, yields $G_{S}=$ $10.21 \mathrm{GeV}^{-2}$. When we compare $G_{P}^{e f f}$ with $G_{S}^{e f f}$, we see that we can expect the resonant enhancement of the spectral functions for the pseudoscalar-isovector channel will be larger in magnitude than that of the spectral function of the scalar-isovector channel. On the other hand, since the 't Hooft interaction does not affect the vector or axial-vector coupling constants, these coupling constants would be equal in our model, leading us to expect quite similar spectral functions for these two channels.

In our study of the $\eta$ mesons we used $G_{V}=13.00 \mathrm{GeV}^{-2}$. However, the results for the $\eta$ mesons are not particularly sensitive to that parameter. In earlier work, with a sharp cutoff for the NJL model of $\Lambda_{3}=0.622 \mathrm{GeV}$, we used $G_{V}=12.46 \mathrm{GeV}^{-2}$ to fit the energy of the $\omega(782)$ [11]. In other calculations we have used $G_{V}=11.46 \mathrm{GeV}^{-2}$. In this work we present the results for the latter value. (We have checked that the spectral functions calculated with either $G_{V}=12.46 \mathrm{GeV}^{-2}$ or $G_{V}=11.46 \mathrm{GeV}^{-2}$ differ by less than 4 percent.)

It was found in our various studies of meson properties, made after we completed Ref. [11], that the use of a sharp cutoff did not allow us to study radial excitations with a large number of nodes. However, we found that a Gaussian regulator, $\exp \left[-\vec{k}^{2} / \alpha^{2}\right]$, with $\alpha=0.605 \mathrm{GeV}$, gave similar results as that of the sharp cutoff $\Lambda_{3}=0.622 \mathrm{GeV}$ for the lowlying states and also allowed us to describe radial excitations with many nodes. Therefore, all our more recent calculations have been made using the Gaussian regulator with $\alpha=0.605$ GeV. (The coupling parameters obtained in Ref.[8], for example, were determined with this Gaussian regulator, so that any change of the regulator parameter, $\alpha$, would require that we provide a new set of coupling parameters corresponding to the new value of $\alpha$.)

We have recently reported results of our calculations of the temperature dependence of the spectra of various meons [6]. These calculations were made using our generalized NJL model which includes a covariant model of confinement. We have presented results for the $\pi, K, a_{0}, f_{0}$ and $K_{0}^{*}$ mesons in Ref.[6]. In that work, temperature-dependent constituent 
quark masses were calculated using the equation [12]

$$
m(T)=m^{0}+2 G_{S}(T) N_{c} \frac{m(T)}{\pi^{2}} \int_{0}^{\Lambda} d p \frac{p^{2}}{E_{p}} \tanh \left(\frac{1}{2} \beta E_{p}\right) .
$$

Here, $m^{0}$ is the current quark mass, $G_{S}(T)$ is a temperature-dependent coupling constant introduced in our model. (In Ref.[6] we used model 1 for $G_{S}(T)$.) Here, $N_{c}=3$ is the number of colors, $\beta=1 / T$ and $E_{p}=\left[\vec{p}^{2}+m^{2}(T)\right]^{1 / 2}$. Further, $\Lambda=0.631 \mathrm{GeV}$ is a cutoff such that $|\vec{p}| \leq \Lambda$. We have put $\Lambda=0.631 \mathrm{GeV}$, since that is the cutoff that is often used when solving Eq. (1.4) [9]. We have also used $m_{u}^{0}=5.5 \mathrm{MeV}$ and $m_{s}^{0}=120 \mathrm{MeV}$. Since in our work dealing with meson spectra, we have used $m_{u}=0.364 \mathrm{GeV}$ and $m_{s}=0.565 \mathrm{GeV}$ as phenomenological parameters, we have put $G_{S}(0)=2 G$, with $G=5.691 \mathrm{GeV}^{-2}$, in the notation of Ref.[12], to reproduce those values. In Ref.[12] $G$ is one-half of the $G_{S}$ defined in Eq.(1.1).

Since the use of temperature-dependent coupling constants is an unusual feature of our work, we now describe some advantages that follow from that choice. Our original introduction of temperature dependence, $G(T)=G\left[1-0.17\left(T / T_{c}\right)\right]$, with $T_{c}=170 \mathrm{MeV}$, had the purpose of simulating the dynamical interactions which eliminate pion or kaon condensation at relatively low temperatures. However, we found when solving Eq.(1.4) for the temperature-dependent constituent mass of the up quark, chiral symmetry was (partially) restored at lower temperatures than when constant coupling parameters were used. This may be seen in Fig.1, where we show the results that follow from the use of a constant value of $G(T)=5.691 \mathrm{GeV}^{-2}$ (in the notation of Ref.[12]) as a dotted line. Here we have used $m^{0}=5.5 \mathrm{MeV}$ as the current quark mass. For the solid and dashed curves in Fig.1 we have used $G(T)=G\left[1-0.17\left(T / T_{c}\right)\right]$. For the sake of this discussion, let us consider the reduction of the constituent mass to $50 \mathrm{MeV}$ from $364 \mathrm{MeV}$ as signal of the (partial) restoration of chiral symmetry. For the dashed and solid curves, that restoration take place at about $T_{c}=170 \mathrm{MeV}$, while for the dotted curve the (partial) restoration of chiral symmetry takes place at about $250 \mathrm{MeV}$. For QCD, the transition temperature is about 150-170 $\mathrm{MeV}$. Since we are attempting to create a model that has some correspondence to QCD, with dynamical quarks, we see that the temperature-dependent coupling constants lead to the desired behavior.

The temperature dependence of the constituent mass in the case of temperaturedependent coupling constants is such that we described the mesonic confinement- 
deconfinement transition as taking place at $T \approx T_{c}$ with $T_{c}=170 \mathrm{MeV}$ in Ref. [6]. In that work we have studied the confinement-deconfinement transition for the $\pi, K, a_{0}, f_{0}$ and $K_{0}^{*}$ mesons. We have checked that, without the introduction of the temperature-dependent cou-

pling constants, the confinement-deconfinement transition would take place at a significantly higher temperature.

A further advantage of the use of our temperature-dependent coupling parameter is that the NJL interaction goes to zero for $T=5.88 T_{c}$. (It is put equal to zero for $T>5.88 T_{c}$.) We suggest that that is consistent with QCD thermodynamics, since it appears that the system is weakly coupled for $T \geq 6 T_{c}[5]$.

In Fig. 2 we show the behavior of both the up and strange quark constituent masses calculated with Eq.(1.4) using current masses $m_{u}^{0}=5.50 \mathrm{MeV}$ and $m_{s}^{0}=120 \mathrm{MeV}$. For the calculations made in this work, we have used the temperature-dependent up (or down) quark masses shown in Fig.2 for temperatures for which the quark masses are quite small. Therefore, our results are insensitive to variations of the mass values shown in Fig.2.

In calculating the constituent mass values we have neglected the confining interaction. That interaction was taken into account in our earlier Euclidean-space calculation of the quark self-energy [13], which also included the effects related to the 't Hooft interaction. We found that, to a good approximation, we could neglect the confining and 't Hooft interactions, if we modified the value of the NJL coupling constant, $G_{S}$, and we adopt that approach when using Eq.(1.4).

The organization of our work is as follows. In Sec.II we review our calculations of polarization functions at finite temperature. In Sec.III we discuss the calculation of hadronic current correlation functions, making use of the results presented in Sec.II. In Sections IV and $\mathrm{V}$ we present the results of our numerical calculations of correlators of pseudoscalar and vector currents, respectively. We also compare our results to some recent lattice calculations of pseudoscalar and vector correlators [14-16]. Finally, in Sec.VI we present same further discussion and conclusions.

\section{POLARIZATION FUNCTIONS AT FINITE TEMPERATURE}

The basic polarization function that is calculated in the NJL model is shown in Fig.3. We will consider calculations of such functions in the frame where $\vec{P}=0$. In our earlier work, 


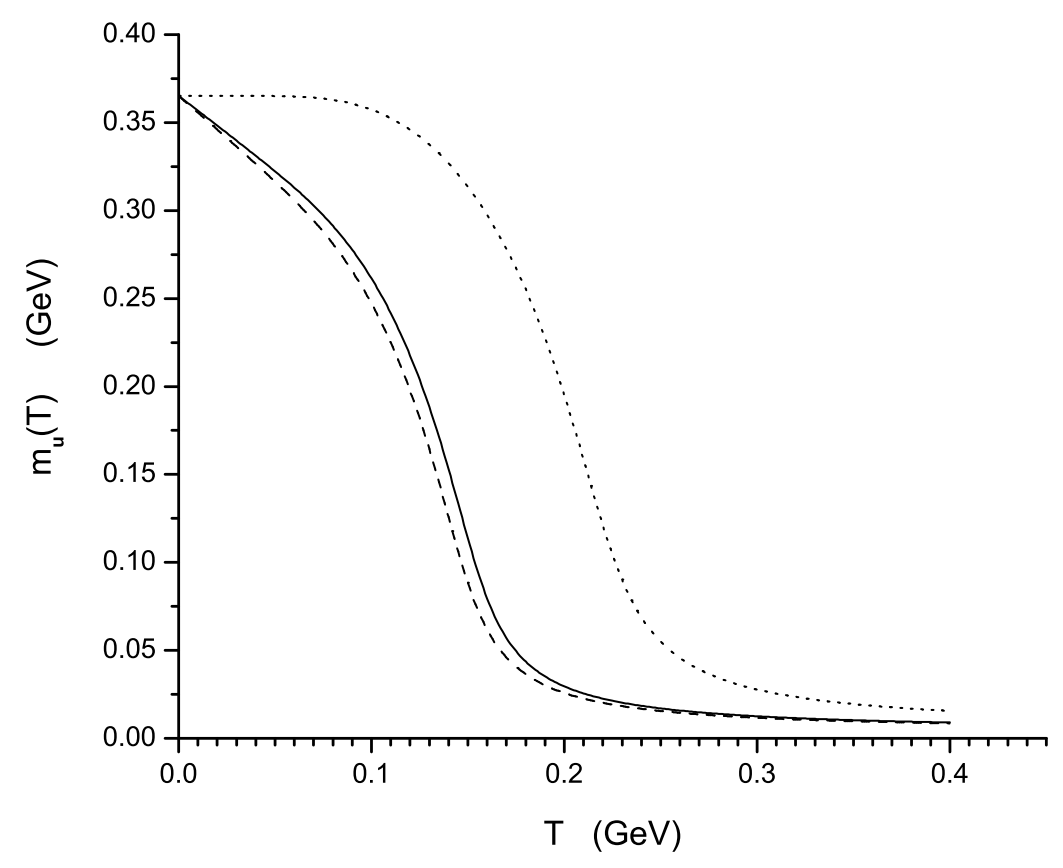

FIG. 1: We exhibit the solution for $m_{u}(T)$ obtained using Eq.(5.38) of Ref.[12], where $m^{0}=5.50$ $\mathrm{MeV}$ and $\Lambda=0.631 \mathrm{GeV}$. The dotted curve corresponds to the use of a constant value $G=5.691$ $\mathrm{GeV}^{-2}$, in the notation of Ref. [12]. For the solid and dashed curves we have used $G(T)=$ $G\left[1-0.17\left(T / T_{c}\right)\right]$. For the solid curve $T_{c}=0.170 \mathrm{GeV}$ and for the dashed curve we have used $T_{c}=0.150 \mathrm{GeV}$.

calculations were made after a confinement vertex was included. That vertex is represented by the filled triangular region in Fig.3. However, we here consider calculations for $T \geq 1.2 T_{c}$ where confinement may be neglected. We will, however, use the temperature-dependent mass values shown in Fig.2.

The procedure we adopt is based upon the real-time finite-temperature formalism, in which the imaginary part of the polarization function may be calculated. Then, the real part of the function is obtained using a dispersion relation. The result we need for this work has been already given in the work of Kobes and Semenoff [17]. (In Ref. [17] the quark momentum in Fig.3 is $k$ and the antiquark momentum is $k-P$. We will adopt that notation in this section for ease of reference to the results presented in Ref. [17].) With reference to 


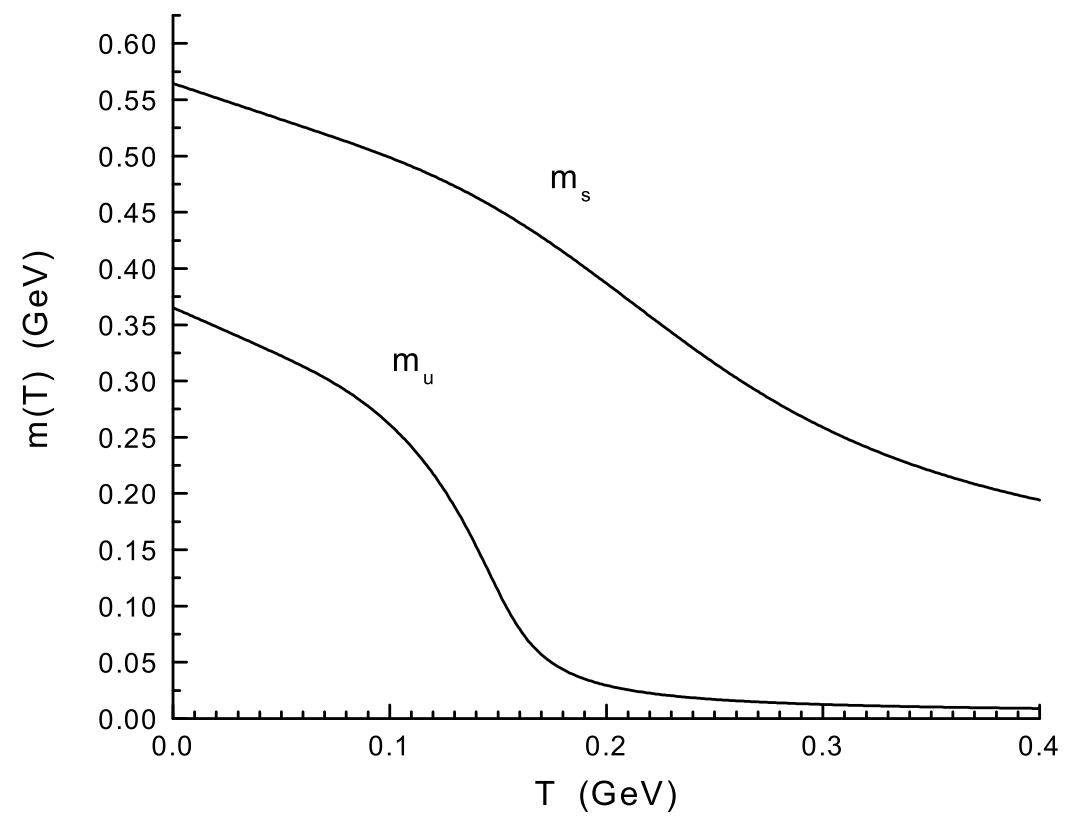

FIG. 2: Temperature dependent constituent mass values, $m_{u}(T)$ and $m_{s}(T)$, calculated in a meanfield approximation [12], are shown. [See Eq. (1.4)]. Here $m_{u}^{0}=0.0055 \mathrm{GeV}, m_{s}^{0}=0.120 \mathrm{GeV}$, and $G(T)=5.691\left[1-0.17\left(T / T_{c}\right)\right]$, if we use Klevansky's notation [12]. (The value of $G_{S}$ of Eq. (1.1) is twice the value of $G$ used in [12].)

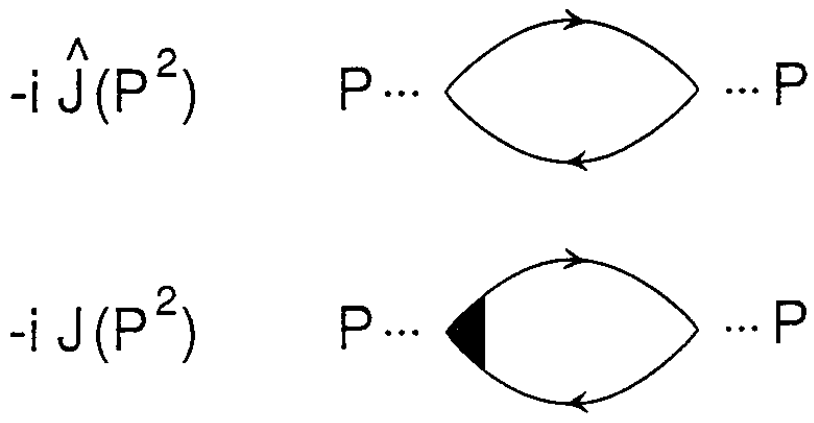

FIG. 3: The upper figure represents the basic polarization diagram of the NJL model in which the lines represent a constituent quark and a constituent antiquark. The lower figure shows a confinement vertex [filled triangular region] used in our earlier work. For the present work we neglect confinement for $T \geq 1.2 T_{c}$, with $T_{c}=150 \mathrm{MeV}$. 
Eq. (5.4) of Ref. [17], we write the imaginary part of the scalar polarization function as

$$
\begin{array}{r}
\operatorname{Im} J_{S}\left(P^{2}, T\right)=\frac{1}{2}\left(2 N_{c}\right) \beta_{S} \epsilon\left(P^{0}\right) \int \frac{d^{3} k}{(2 \pi)^{3}} e^{-\vec{k}^{2} / \alpha^{2}}\left(\frac{2 \pi}{2 E_{1}(k) 2 E_{2}(k)}\right) \\
\left\{\left(1-n_{1}(k)-n_{2}(k)\right) \delta\left(P^{0}-E_{1}(k)-E_{2}(k)\right)\right. \\
-\left(n_{1}(k)-n_{2}(k)\right) \delta\left(P^{0}+E_{1}(k)-E_{2}(k)\right) \\
-\left(n_{2}(k)-n_{1}(k)\right) \delta\left(P^{0}-E_{1}(k)+E_{2}(k)\right) \\
\left.-\left(1-n_{1}(k)-n_{2}(k)\right) \delta\left(P^{0}+E_{1}(k)+E_{2}(k)\right)\right\} .
\end{array}
$$

Here, $E_{1}(k)=\left[\vec{k}^{2}+m_{1}^{2}(T)\right]^{1 / 2}$. Relative to Eq. (5.4) of Ref.[17], we have changed the sign, removed a factor of $g^{2}$ and have included a statistical factor of $2 N_{c}$, where the factor of 2 arises from the flavor trace. In addition, we have included a Gaussian regulator, $\exp \left[-\vec{k}^{2} / \alpha^{2}\right]$, with $\alpha=0.605 \mathrm{GeV}$, which is the same as that used in most of our applications of the NJL model in the calculation of meson properties. We also note that

$$
n_{1}(k)=\frac{1}{e^{\beta E_{1}(k)}+1},
$$

and

$$
n_{2}(k)=\frac{1}{e^{\beta E_{2}(k)}+1}
$$

For the calculation of the imaginary part of the polarization function, we may put $k^{2}=$ $m_{1}^{2}(T)$ and $(k-P)^{2}=m_{2}^{2}(T)$, since in that calculation the quark and antiquark are onmass-shell. In Eq. (2.1) the factor $\beta_{S}$ arises from a trace involving Dirac matrices, such that

$$
\begin{aligned}
\beta_{S} & =-\operatorname{Tr}\left[\left(\not k+m_{1}\right)\left(\not k-\not P+m_{2}\right)\right] \\
& =2 P^{2}-2\left(m_{1}+m_{2}\right)^{2},
\end{aligned}
$$

where $m_{1}$ and $m_{2}$ depend upon temperature. In the frame where $\vec{P}=0$, and in the case $m_{1}=m_{2}$, we have $\beta_{S}=2 P_{0}^{2}\left(1-4 m^{2} / P_{0}^{2}\right)$. For the scalar case, with $m_{1}=m_{2}$, we find

$$
\operatorname{Im} J_{S}\left(P^{2}, T\right)=\frac{N_{c} P_{0}^{2}}{4 \pi}\left(1-\frac{4 m^{2}}{P_{0}^{2}}\right)^{3 / 2} e^{-\vec{k}^{2} / \alpha^{2}}\left[1-2 n_{1}(k)\right],
$$

where

$$
\vec{k}^{2}=\frac{P_{0}^{2}}{4}-m^{2}(T)
$$


For pseudoscalar mesons, we replace $\beta_{S}$ by

$$
\begin{aligned}
\beta_{P} & =-\operatorname{Tr}\left[i \gamma_{5}\left(\not k+m_{1}\right) i \gamma_{5}\left(\not k-\not p+m_{2}\right)\right] \\
& =2 P^{2}-2\left(m_{1}-m_{2}\right)^{2}
\end{aligned}
$$

which for $m_{1}=m_{2}$ is $\beta_{P}=2 P_{0}^{2}$ in the frame where $\vec{P}=0$. We find, for the $\pi$ mesons,

$$
\operatorname{Im} J_{P}\left(P^{2}, T\right)=\frac{N_{c} P_{0}^{2}}{4 \pi}\left(1-\frac{4 m(T)^{2}}{P_{0}^{2}}\right)^{1 / 2} e^{-\vec{k}^{2} / \alpha^{2}}\left[1-2 n_{1}(k)\right],
$$

where $\vec{k}^{2}=P_{0}^{2} / 4-m_{u}^{2}(T)$, as above. Thus, we see that, relative to the scalar case, the phase space factor has an exponent of $1 / 2$ corresponding to a $s$-wave amplitude. For the scalars, the exponent of the phase-space factor is 3/2, as seen in Eq. (2.6).

For a study of the vector-isovector correlators, we introduce conserved vector currents $j_{\mu, i}(x)=\tilde{q}(x) \gamma_{\mu} \lambda_{i} q(x)$ with $\mathrm{i}=1,2$ and 3 . In this case we define

$$
J_{V}^{\mu \nu}\left(P^{2}, T\right)=\left(g^{\mu \nu}-\frac{P^{\mu} P^{\nu}}{P^{2}}\right) J_{V}\left(P^{2}, T\right)
$$

and

$$
C_{V}^{\mu \nu}\left(P^{2}, T\right)=\left(g^{\mu \nu}-\frac{P^{\mu} P^{\nu}}{P^{2}}\right) C_{V}\left(P^{2}, T\right),
$$

taking into account the fact that the current $j_{\mu, i}(x)$ is conserved. We may then use the fact that

$$
J_{V}\left(P^{2}, T\right)=\frac{1}{3} g_{\mu \nu} J_{V}^{\mu \nu}\left(P^{2}, T\right)
$$

and

$$
\begin{aligned}
\operatorname{Im} J_{V}\left(P^{2}, T\right) & =\frac{2}{3}\left[\frac{P_{0}^{2}+2 m_{u}^{2}(T)}{4 \pi}\right]\left(1-\frac{4 m_{u}^{2}(T)}{P_{0}^{2}}\right)^{1 / 2} e^{-\vec{k}^{2} / \alpha^{2}}\left[1-2 n_{1}(k)\right] \\
& \simeq \frac{2}{3} \operatorname{Im} J_{P}\left(P^{2}, T\right) .
\end{aligned}
$$

We now consider

$$
\beta_{\mu \nu}^{V}=\operatorname{Tr}\left[\gamma_{\mu}\left(\not k+m_{1}\right) \gamma_{\nu}\left(\not k-\not P+m_{2}\right)\right]
$$

and calculate

$$
g^{\mu \nu} \beta_{\mu \nu}^{V}=4\left[P^{2}-m_{1}^{2}-m_{2}^{2}+4 m_{1} m_{2}\right]
$$


which, in the equal-mass case, is equal to $4 P_{0}^{2}+8 m^{2}(T)$, when $m_{1}=m_{2}$ and $\vec{P}=0$. This result will be needed when we calculate the correlator of vector currents in the next section. Note that for the elevated temperatures considered in this work $m_{u}(T)=m_{d}(T)$ is quite small, so that $4 P_{0}^{2}+8 m_{u}^{2}(T)$ can be approximated by $4 P_{0}^{2}$ when we consider the vector current correlation functions. In that case, we have

$$
\operatorname{Im} J_{V}\left(P^{2}, T\right) \simeq \frac{2}{3} \operatorname{Im} J_{P}\left(P^{2}, T\right)
$$

At this point it is useful to define functions that do not contain the Gaussian regulator:

$$
\operatorname{Im} \tilde{J}_{P}\left(P^{2}, T\right)=\frac{N_{c} P_{0}^{2}}{4 \pi}\left(1-\frac{4 m(T)^{2}}{P_{0}^{2}}\right)^{1 / 2}\left[1-2 n_{1}(k)\right],
$$

and

$$
\operatorname{Im} \tilde{J}_{V}\left(P^{2}, T\right)=\frac{2}{3} \frac{N_{c} P_{0}^{2}}{4 \pi}\left(1-\frac{4 m(T)^{2}}{P_{0}^{2}}\right)^{1 / 2}\left[1-2 n_{1}(k)\right] .
$$

For the functions defined in Eq. (2.19) and (2.20) we need to use a twice-subtracted dispersion relation to obtain $\operatorname{Re} \tilde{J}_{P}\left(P^{2}, T\right)$, or $\operatorname{Re} \tilde{J}_{V}\left(P^{2}, T\right)$. For example,

$$
\begin{array}{r}
\operatorname{Re} \tilde{J}_{P}\left(P^{2}, T\right)=\operatorname{Re} \tilde{J}_{P}(0, T)+\frac{P^{2}}{P_{0}^{2}}\left[\operatorname{Re} \tilde{J}_{P}\left(P_{0}^{2}, T\right)-\operatorname{Re} \tilde{J}_{P}(0, T)\right]+ \\
\frac{P^{2}\left(P^{2}-P_{0}^{2}\right)}{\pi} \int_{m^{2}(T)}^{\tilde{\Lambda}^{2}} d s \frac{\operatorname{Im} \tilde{J}_{P}(s, T)}{s\left(P^{2}-s\right)\left(P_{0}^{2}-s\right)},
\end{array}
$$

where $\tilde{\Lambda}^{2}$ can be quite large since the integral over the imaginary part of the polarization function is now convergent. We may introduce $\tilde{J}_{P}\left(P^{2}, T\right)$ and $\tilde{J}_{V}\left(P^{2}, T\right)$ as complex functions, since we now have both the real and imaginary parts of these functions. We note that the construction of either $\operatorname{Re} J_{P}\left(P^{2}, T\right)$ or $\operatorname{Re} J_{V}\left(P^{2}, T\right)$ by means of a dispersion relation does not require a subtraction. We use these functions to define the complex functions $J_{P}\left(P^{2}, T\right)$ and $J_{V}\left(P^{2}, T\right)$.

In order to make use of Eq.(2.21) we need to specify $\operatorname{Re} \tilde{J}_{P}(0)$ and $\operatorname{Re} \tilde{J}_{P}\left(P_{0}^{2}\right)$. We found it useful to take $P_{0}^{2}=-1.0 \mathrm{GeV}^{2}$ and to put $\operatorname{Re} \tilde{J}_{P}(0)=\operatorname{Re} J_{P}(0)$ and $\operatorname{Re} \tilde{J}_{P}\left(P_{0}^{2}\right)=\operatorname{Re} J_{P}\left(P_{0}^{2}\right)$. The quantities $\operatorname{Re} \tilde{J}_{V}(0)$ and $\operatorname{Re} \tilde{J}_{V}\left(P_{0}^{2}\right)$ are determined in an analogous function. This procedure in which we fix the behavior of a function such as $\tilde{J}_{P}\left(P^{2}\right)$ or $\tilde{J}_{V}\left(P^{2}\right)$, which may be used when making calculations for large $P^{2}$, is quite analogous to the procedure used in Ref. [18]. In that work we made use of dispersion relations to construct a continuous vector-isovector current correlation function that had the correct perturbative behavior for $P^{2} \rightarrow-\infty$ and 
also described the low-energy resonance present in the correlator due to the excitation of the $\rho$ meson. In Ref. [18] the NJL model was shown to provide a quite satisfactory description of the low-energy resonant behavior of the vector-isovector correlation function.

We may compare our expressions for $(1 / \pi) \operatorname{Im} J\left(P^{2}\right)$ with those given in Eq.(2.7) of Ref.[19]. We find that the scalar and pseudoscalar polarization functions are defined there with a sign opposite to ours. However, since we have defined the pseudoscalar current using the matrix $i \gamma_{5}$ rather than $\gamma_{5}$, which was used in Ref.[19], our expression for the spectral function agrees with that of Ref.[19] with respect to the sign in the pseudoscalar

case. In the case of the vector and axial-vector spectral functions, we have the same sign convention as that of Ref.[19]. We disagree with the expressions for the spectral functions given for the scalar and axial-vector currents in Ref.[19] with respect to the exponent of the phase-space factor. In Ref.[19], the phase-space factor for the scalar and axial-vector case is $\left[1-(2 m / \omega)^{2}\right]^{1 / 2}$, while we have $\left[1-(2 m / \omega)^{2}\right]^{3 / 2}$ for those spectral functions. We agree that the phase-space factor is $\left[1-(2 m / \omega)^{2}\right]^{1 / 2}$ for the pseudoscalar and vector current spectral functions.

In Ref.[19] use is made of the hard-thermal-loop (HTL) approximation. It was found that the scalar channels are only moderately influenced by the HTL medium effects, while the HTL vertex corrections lead to divergent vector correlators.

\section{CALCULATION OF HADRONIC CURRENT CORRELATION FUNCTIONS}

In this section we consider the calculation of temperature-dependent hadronic current correlation functions. The general form of the correlator is a transform of a time-ordered product of currents,

$$
i C\left(P^{2}, T\right)=\int d^{4} x e^{i P \cdot x} \ll T(j(x) j(0)) \gg,
$$

where the double bracket is a reminder that we are considering the finite-temperature case.

For the study of pseudoscalar states, we may consider currents of the form $j_{P, i}(x)=$ $\tilde{q}(x) i \gamma_{5} \lambda^{i} q(x)$, where, in the case of the $\pi$ mesons, $i=1,2$ and 3 . For the study of scalarisoscalar mesons, we introduce $j_{S, i}(x)=\tilde{q}(x) \lambda^{i} q(x)$, with $i=0$ for the flavor-singlet current and $i=8$ for the flavor-octet current [7].

In the case of the pseudoscalar-isovector mesons, the correlator may be expressed in terms 
of the basic vacuum polarization function of the NJL model, $J_{P}\left(P^{2}, T\right)[9,10,12]$. Thus,

$$
C_{P}\left(P^{2}, T\right)=J_{P}\left(P^{2}, T\right) \frac{1}{1-G_{P}(T) J_{P}\left(P^{2}, T\right)},
$$

where $G_{P}(T)$ is the coupling constant appropriate for our study of $\pi$ mesons. We have found $G_{P}(T)=13.49 \mathrm{GeV}^{-2}$ by fitting the pion mass in a calculation made at $T=0$, with $m_{u}=m_{d}=0.364 \mathrm{GeV}$. The result given in Eq. (3.2) is only expected to be useful for small $P^{2}$, since the Gaussian regulator strongly modifies the large $P^{2}$ behavior. Therefore, we suggest that the following form is useful, if we are to consider the larger values of $P^{2}$

$$
\frac{C_{P}\left(P^{2}, T\right)}{P^{2}}=\left[\frac{\tilde{J}_{P}\left(P^{2}, T\right)}{P^{2}}\right] \frac{1}{1-G_{P}(T) J_{P}\left(P^{2}, T\right)} .
$$

(As usual, we put $\vec{P}=0$.) This form has two important features. At large $P_{0}^{2}$, $\operatorname{Im} C_{P}\left(P_{0}, T\right) / P_{0}^{2}$ is a constant, since $\operatorname{Im} \tilde{J}_{P}\left(P_{0}^{2}, T\right)$ is proportional to $P_{0}^{2}$. Further, the denominator of the second term on the right-hand side of Eq.(3.3) goes to 1 for large $P_{0}^{2}$. On the other hand, at small $P_{0}^{2}$, that denominator is capable of describing resonant enhancement of the correlation function. (We may again refer to Ref. [18], in which a similar approximation is described.)

We then have

$$
C_{V}\left(P^{2}, T\right)=\tilde{J}_{V}\left(P^{2}, T\right) \frac{1}{1-G_{V}(T) J_{V}\left(P^{2}, T\right)}
$$

where we have introduced

$$
\begin{aligned}
\operatorname{Im} \tilde{J}_{V}\left(P^{2}, T\right) & =\frac{2}{3}\left[\frac{P_{0}^{2}+2 m_{u}^{2}(T)}{4 \pi}\right]\left(1-\frac{4 m_{u}^{2}(T)}{P_{0}^{2}}\right)^{1 / 2}\left[1-2 n_{1}(k)\right] \\
& \simeq \frac{2}{3} \operatorname{Im} \tilde{J}_{P}\left(P^{2}, T\right) .
\end{aligned}
$$

(See Eq.(2.7) for the specification of $k=|\vec{k}|$.) In the literature, $\omega$ is used instead of $P_{0}[14$ -16]. We may define the spectral functions

$$
\sigma_{V}(\omega, T)=\frac{1}{\pi} \operatorname{Im} C_{V}(\omega, T)
$$

and

$$
\sigma_{P}(\omega, T)=\frac{1}{\pi} \operatorname{Im} C_{P}(\omega, T)
$$


We may use the notation $\bar{\sigma}_{P}(\omega, T)$ and $\bar{\sigma}_{V}(\omega, T)$ for the spectral functions given in the literature [14-16]. We have the following relations:

$$
\bar{\sigma}_{P}(\omega, T)=\sigma_{P}(\omega, T)
$$

and

$$
\frac{\bar{\sigma}_{V}(\omega, T)}{2}=\frac{3}{4} \sigma_{V}(\omega, T)
$$

where the factor of $3 / 4$ arises because, in Refs. [14-16], there is a division by 4 , while we have divided by 3 as in Eq. (2.13).

In addition to providing values of $\sigma_{V}(\omega, T) / \omega^{2}$ and $\sigma_{P}(\omega, T) / \omega^{2}$, the $\tau$-dependent hadronic correlators $G_{P}(\tau, T)$ and $G_{V}(\tau, T)$ were introduced [14-16], with

$$
G(\tau, T)=\int_{0}^{\infty} d \omega \sigma(\omega, T) K(\omega, \tau, T) .
$$

Here,

$$
K(\omega, \tau, T)=\frac{\cosh \left[\omega\left(\tau-\frac{1}{2 T}\right)\right]}{\sinh \left(\frac{\omega}{2 T}\right)} .
$$

We will present results of our calculations of $G_{P}(\tau, T)$ and $G_{V}(\tau, T)$ in Sections IV and $\mathrm{V}$, respectively. We will also present the values obtained for $\operatorname{Im} C_{P}\left(P_{0}, T\right) / P_{0}^{2}$ and $\operatorname{Im} C_{V}\left(P_{0}, T\right) / P_{0}^{2}$ for various values of $T / T_{c}$ in Sections IV and $\mathrm{V}$.

We note that values have been obtained for the spectral functions in the scalar, vector, pseudoscalar and axial-vector channels in Ref.[20]. These functions exhibit resonant features at about 2,7 and $14 \mathrm{GeV}$ when $T \approx 1.4 T_{c}$. High-energy resonances are still present at 5 and $13 \mathrm{GeV}$, when $T \approx 1.9 T_{c}$. The interpretation of these high-energy resonances is uncertain $[21]$.

\section{CORRELATION FUNCTIONS FOR PSEUDOSCALAR-ISOVECTOR CUR- RENTS - NUMERICAL RESULTS}

In Fig.4 we show $\operatorname{Im} C_{P}\left(P_{0}, T\right) / P_{0}^{2}$ for $T / T_{c}=1.5$, and for model 1 , as a solid line. The dashed line represents the result at $T / T_{c}=1.5$, if we put $G_{P}(T)=0$. The results for a broader range of temperatures are shown in Fig.5. In Fig.6 we show the ratios $R_{P}\left(P_{0}, T\right)=$ $\operatorname{Im} C_{P}\left(P_{0}, T\right) / \operatorname{Im} C_{P}^{(0)}\left(P_{0}, T\right)$ calculated for various temperatures. Here, $\operatorname{Im} C_{P}^{(0)}\left(P_{0}, T\right) / P_{0}^{2}$ is 


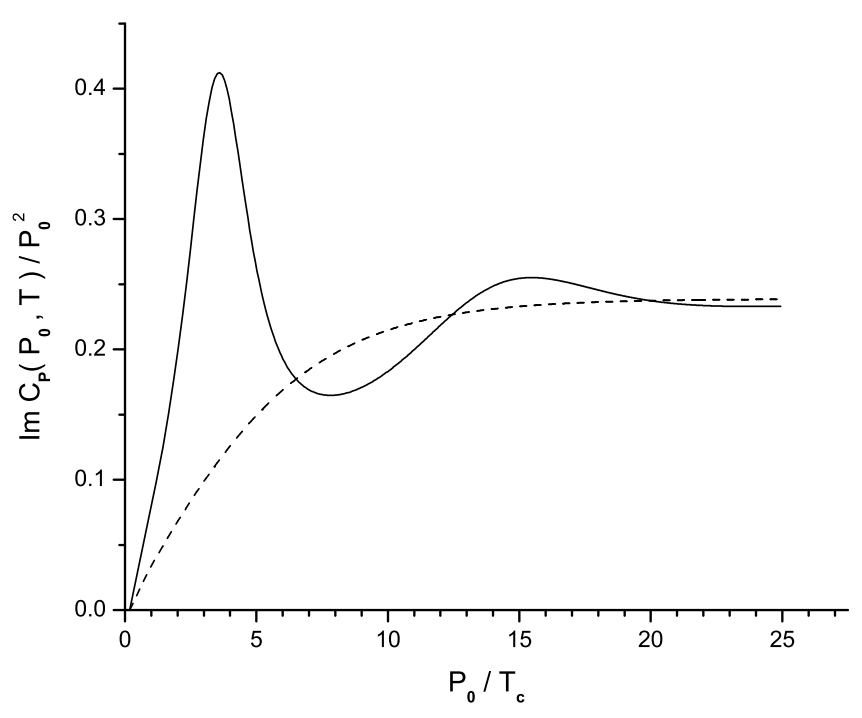

FIG. 4: Values of $\operatorname{Im} C_{P}\left(P_{0}, T\right) / P_{0}^{2}$ are shown at $T / T_{c}=1.5$ for model 1 , where $G_{P}(T)=G_{P}[1-$ $\left.0.17\left(T / T_{c}\right)\right]$ (solid line). The dashed line represents the result obtained when $T / T_{c}=1.5$ and $G_{P}(T)=0$. (Here, the dashed line represents the values of $\operatorname{Im} \tilde{J}_{P}\left(P_{0}, T\right) / P_{0}^{2}$ for $T / T_{c}=1.5$.)

calculated with $G_{P}(T)=0$. In Fig.7 we show the ratio $R_{G}^{(P)}(\tau, T)=G_{P}(\tau, T) / G_{P}^{(0)}(\tau, T)$ for various temperatures. Here, $G_{P}^{(0)}(\tau, T)$ represents $G_{P}(\tau, T)$ evaluated for $G_{P}(T)=0$. In Fig.8 we exhibit $\operatorname{Im} C_{P}\left(P_{0}, T\right) / P_{0}^{2}$ for various temperature for model 2.

In Fig.9 we show, as a dashed line, values of $\operatorname{Im} C_{P}\left(P_{0}, T\right) / P_{0}^{2}$ at $T / T_{c}=5.88$ obtained using a constant value of $G_{P}(T)=13.49 \mathrm{GeV}^{-2}$. The solid line is the result for model 1 (or model 2) at $T / T_{c}=5.88$, in which case $G_{P}(T)=0$ for both models. Since we have argued that for $T / T_{c} \sim 6$ the quark-gluon plasma should be a weakly interacting system, the use of a constant value for $G_{P}(T)$ appears to be unacceptable. We present this result to support our argument that the coupling parameters of the chiral Lagrangian model should be made temperature dependent to be consistent with QCD thermodynamics.

In Fig.10 we show values of $G_{P}(\tau, T) / T^{3}$ for $T / T_{c}=1.5$ [dashed line], $T / T_{c}=3.0$ [dashdotted line] and $T / T_{c}=5.88$ [solid line]. In Fig.1 of Ref. [11] we see a marked difference in the behavior of $G_{P}(\tau, T) / T^{3}$ and $G_{V}(\tau, T) / T^{3}$. (We will present some discussion of this matter in Sec.V.) 


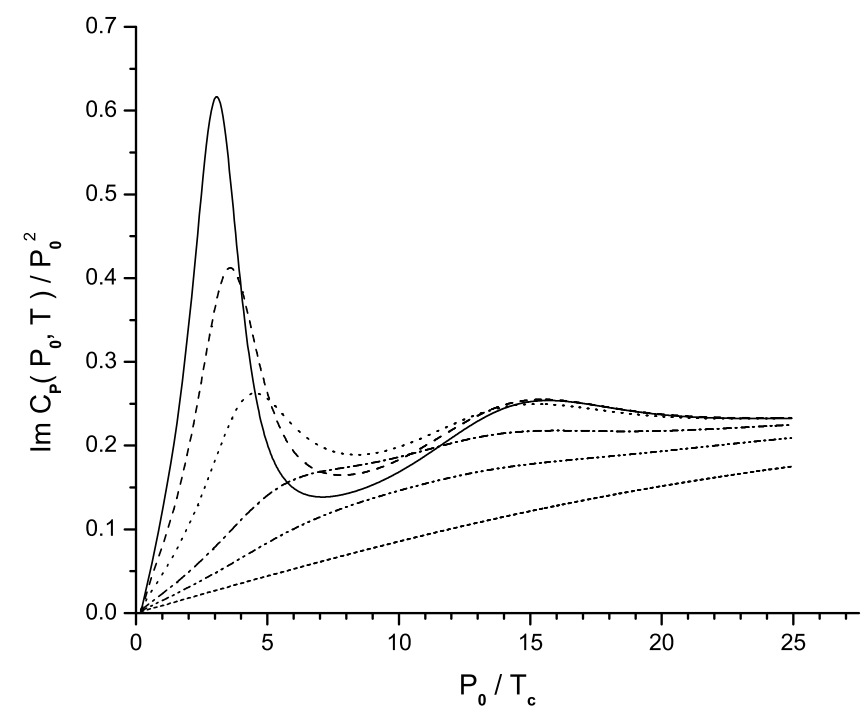

FIG. 5: Values of $\operatorname{Im} C_{P}\left(P_{0}, T\right) / P_{0}^{2}$ are shown for model 1 and for various temperatures: $T / T_{c}=1.2$ [sold line], $T / T_{c}=1.5$ [dashed line], $T / T_{c}=2.0$ [dotted line], $T / T_{c}=3.0$ [dashed-dotted line], $T / T_{c}=4.0$ [dashed-double dotted line], and $T / T_{c}=5.88$ [short-dashed line].

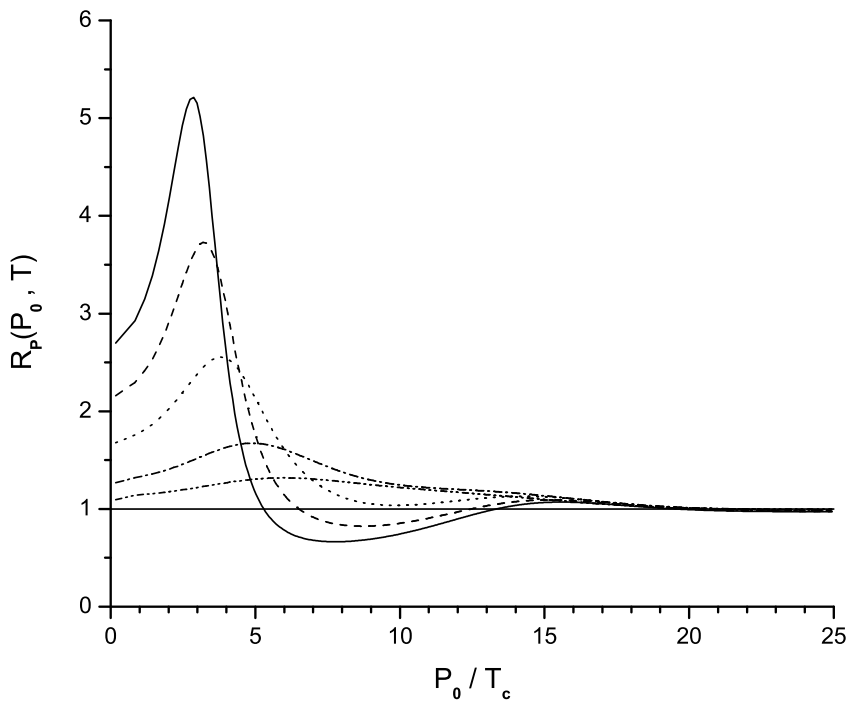

FIG. 6: The ratio $R_{P}\left(P_{0}, T\right)=\operatorname{Im} C_{P}\left(P_{0}, T\right) / \operatorname{Im} C_{P}^{(0)}\left(P_{0}, T\right)$ is shown for various values of $T / T_{c}=$ 1.2 [solid line], $T / T_{c}=1.5$ [dashed line], $T / T_{c}=2.9$ [dotted line], $T / T_{c}=3.0$ [dot-dashed line] and $T / T_{c}=4.0$ [double dot-dashed line]. Here, $\operatorname{Im} C_{P}^{(0)}\left(P_{0}, T\right)$ is calculated at temperature $T$ with $G_{P}(T)=0$. 


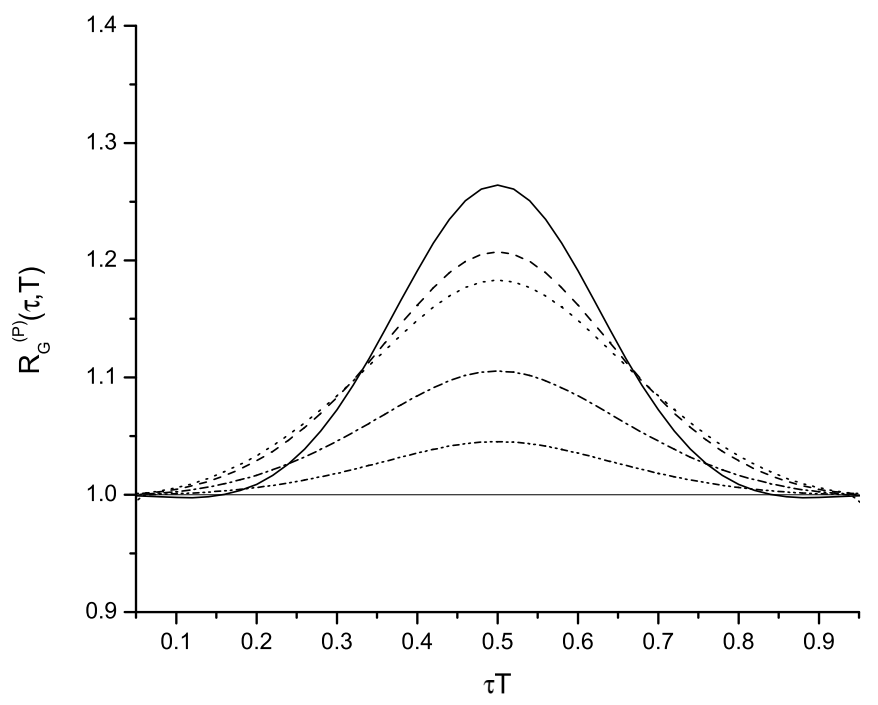

FIG. 7: Values of $R_{G}^{(P)}(\tau, T)$ are shown for values of $T / T_{c}=1.2$ [solid line], $T / T_{c}=1.5$ [dashed line], $T / T_{c}=2.0$ [dotted line], $T / T_{c}=3.0$ [dashed-dotted line], and $T / T_{c}=4.0$ [dashed-double dotted line]. For $T / T_{c}=5.88, R_{G}^{(P)}(\tau, T)=1$.

\section{CORRELATION FUNCTIONS FOR VECTOR-ISOVECTOR CURRENTS - NUMERICAL RESULTS}

In Fig.11 we show the values of $\operatorname{Im} C_{V}\left(P_{0}, T\right) / P_{0}^{2}$ for model 1 and for $T / T_{c}=1.5$, as a solid line. The result for $T / T_{c}=1.5$ and $G_{V}(T)=0$ is represented by the dashed line. [See Fig.4.] In Fig.12 we show values of $\operatorname{Im} C_{V}\left(P_{0}, T\right) / P_{0}^{2}$ for various values of $T / T_{c}$ and for model 1. Corresponding results for model 2 are given in Fig.13.

In Fig.14 we show the ratio $R_{V}\left(P_{0}, T\right)=\operatorname{Im} C_{V}\left(P_{0}, T\right) / \operatorname{Im} C_{V}^{(0)}\left(P_{0}, T\right)$, where $\operatorname{Im} C_{V}^{(0)}\left(P_{0}, T\right)$ is calculated with $G_{V}(T)=0$. (See Fig.9). In Fig.15 we show the ratio $R_{G}^{(V)}(\tau, T)=G_{V}(\tau, T) / G_{V}^{(0)}(\tau, T)$, where $G_{V}(0)(\tau, T)$ represents $G_{V}(\tau, T)$ calculated with $G_{V}(T)=0$. In Fig.16 we show, as a dashed line, values for $T / T_{c}=5.88$ of $\operatorname{Im} C_{V}\left(P_{0}, T\right) / P_{0}^{2}$, which were calculated with a constant value of $G_{V}(T)=11.46 \mathrm{GeV}^{-2}$. The solid line represents the results for $G_{V}(T)=0$, which is characteristic of models 1 and 2 when $T / T_{c}=5.88$. The comments made with respect to Fig.9 are also applicable here.

In Fig.17 we show values of $G_{V}(\tau, T) / T^{3}$ for $T / T_{c}=1.5$ [dashed line], $T / T_{c}=3.0$ [dashdotted line] and $T / T_{c}=5.88$ [solid line]. These results were obtained with model 1, and may be compared to those shown in Fig.9, where we see generally similar behavior. That 


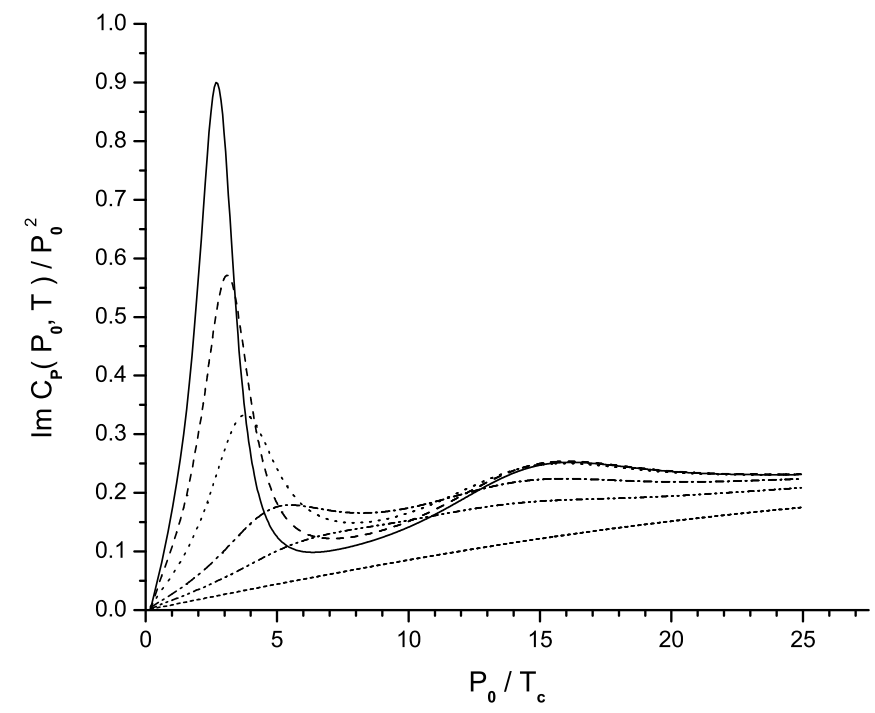

FIG. 8: Values of $\operatorname{Im} C_{P}\left(P_{0}, T\right) / P_{0}^{2}$ are shown at for various temperatures for model 2, with $G_{P}(T)=G_{P}\left[1-0.0289\left(T / T_{c}\right)^{2}\right]$. (See the caption to Fig.5.)

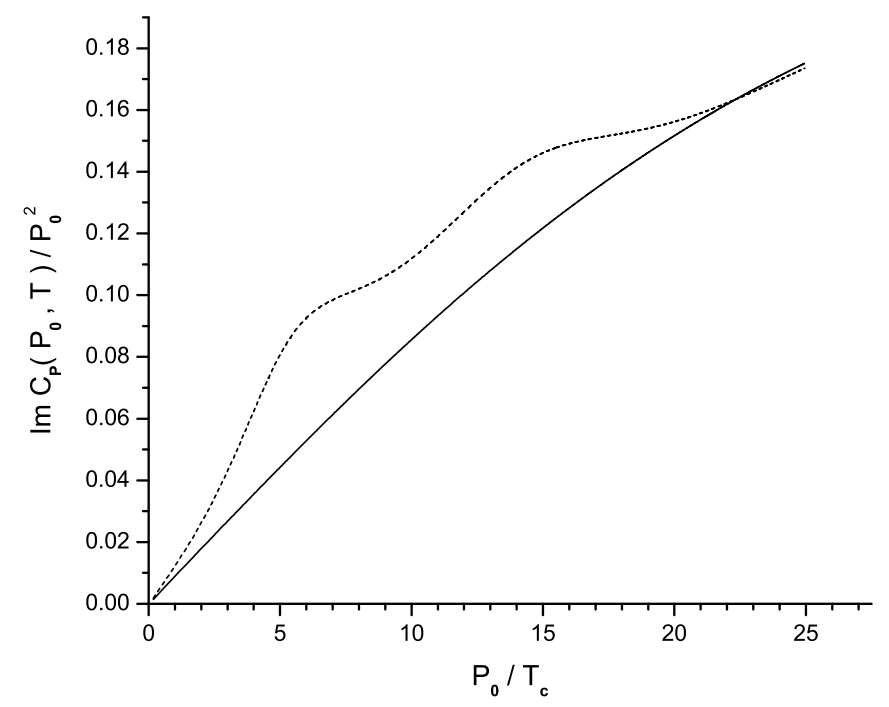

FIG. 9: Values of $\operatorname{Im} C_{P}\left(P_{0}, T\right) / P_{0}^{2}$ are shown for $T / T_{c}=5.88$. The dashed line represents the result when $G_{P}(T)=G_{P}=13.49 \mathrm{GeV}^{-2}$. The solid line is the result for $G_{P}(T)=0$, which is characteristic of models 1 and 2 , when $T / T_{c}=5.88$. (The solid line, therefore, represents the values of $\operatorname{Im} \tilde{J}_{P}\left(P_{0}, T\right) / P_{0}^{2}$ for $T / T_{c}=5.88$.) 


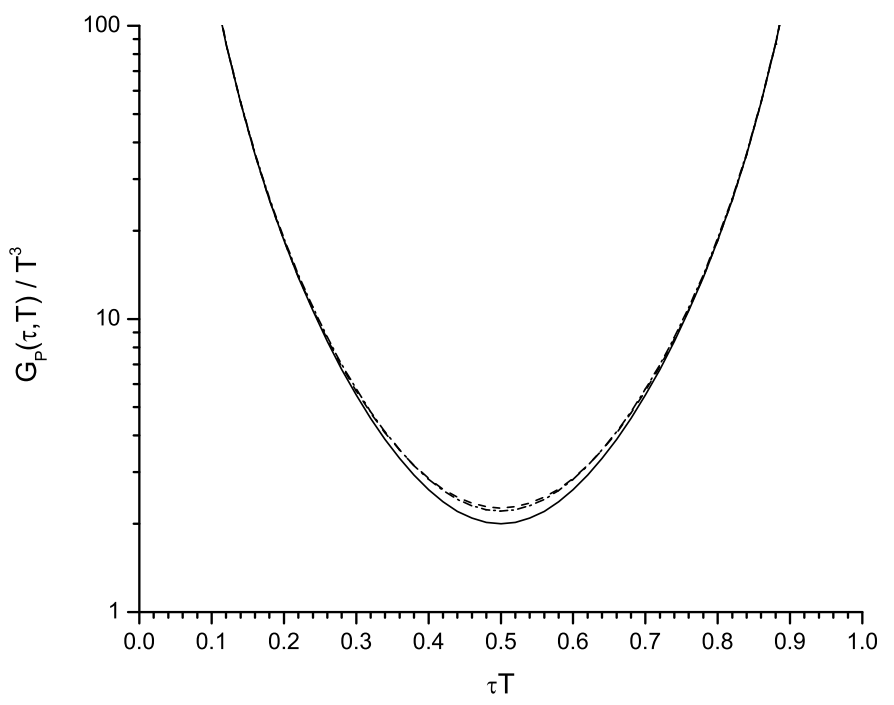

FIG. 10: Values of $G_{P}(\tau, T) / T^{3}$ are shown for $T / T_{c}=1.5$ [dashed line], $T / T_{c}=3.0$ [dashed-dotted line], and $T / T_{c}=5.88$ [solid line]. These results were calculated with model 1.

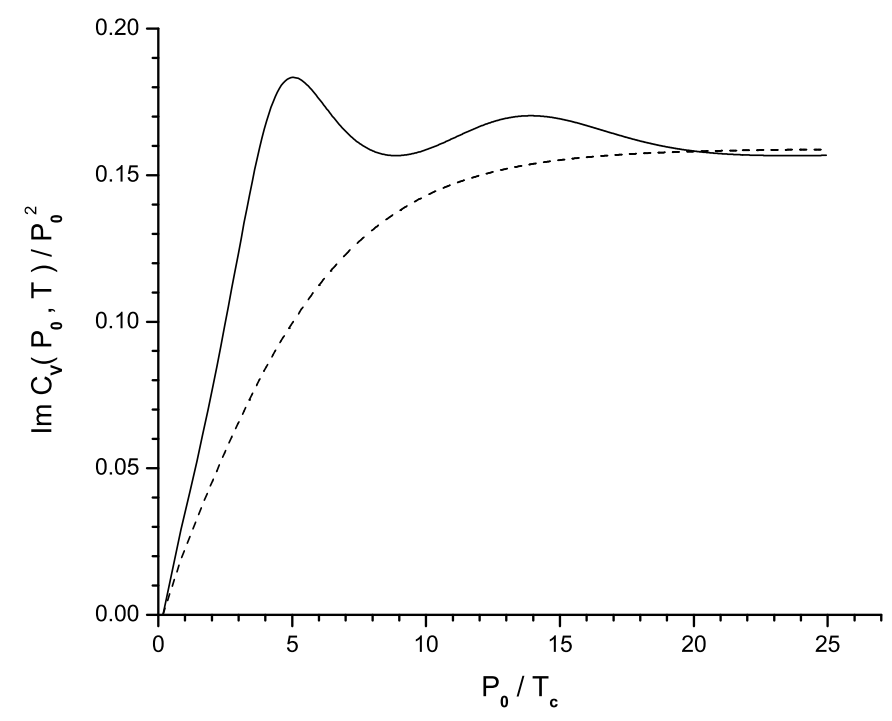

FIG. 11: Values of $\operatorname{Im} C_{V}\left(P_{0}, T\right) / P_{0}^{2}$ are shown for $T / T_{c}=1.5$. (See the caption of Fig.4.)

is in strong contrast to the results shown in Ref. [15], where $G_{V}(\tau, T) / T^{3}$ and $G_{P}(\tau, T) / T^{3}$ shows quite different behavior, with the result for the vector correlator close to the values for $G(T)=0$ at $T / T_{c}=1.5$ and $T / T_{c}=3.0$. This suggests that the value of $G_{V}=11.46$ $\mathrm{GeV}^{-2}$ that we have used in this work may be too large, or that the temperature dependence of $G_{V}(T)$ is such as to yield smaller values than those obtained in this work for model 1 


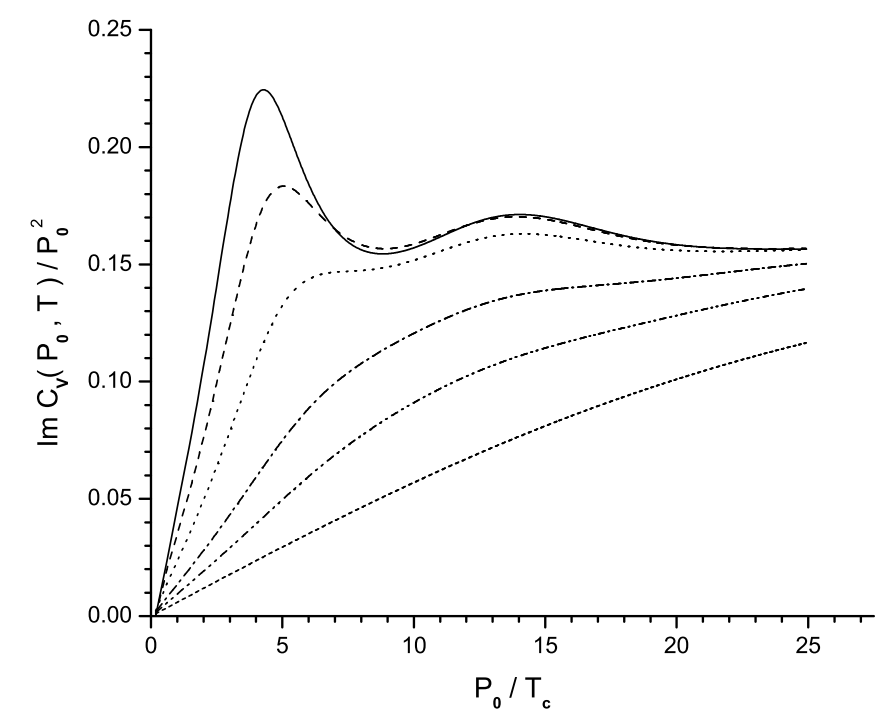

FIG. 12: Values of $\operatorname{Im} C_{V}\left(P_{0}, T\right) / P_{0}^{2}$ are shown for model 1 and for various temperatures. (See the caption of Fig.5.)

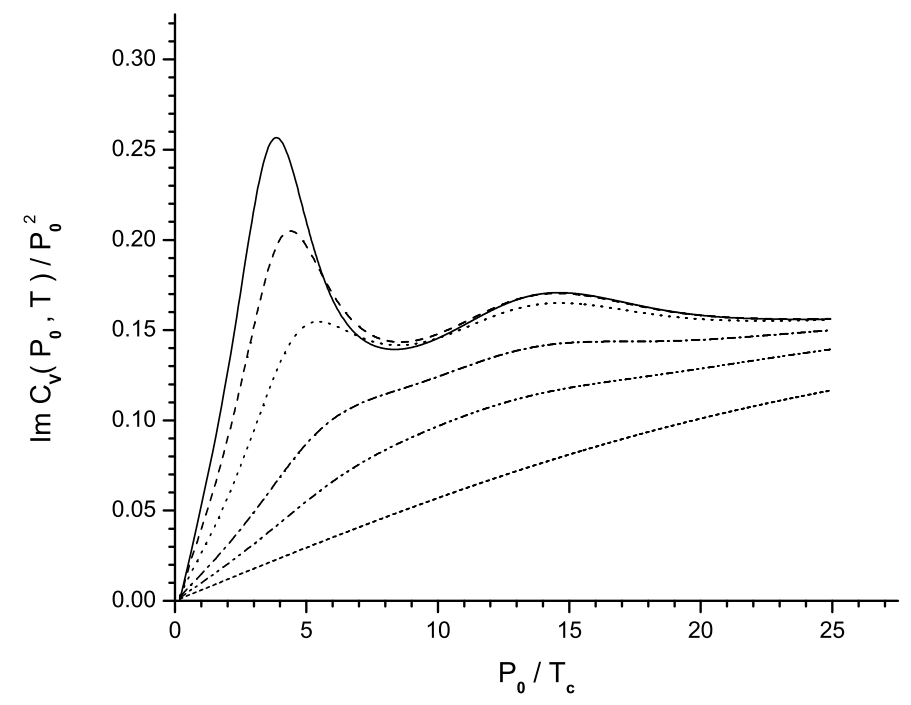

FIG. 13: Values of $\operatorname{Im} C_{V}\left(P_{0}, T\right) / P_{0}^{2}$ are shown for model 2 and for various temperatures. (See the caption of Fig.5.)

or model 2. (In general, we prefer the the second of these possibilities, since our value of $G_{V}$ at $T=0$ is set by fitting meson spectra.) In Fig.18 we show $\operatorname{Im} C_{V}\left(P_{0}, T\right) / P_{0}^{2}$ for $T=1.5 T_{c}$, for model 1, with $G_{V}=11.46 \mathrm{GeV}^{-2}$ [solid line], $G_{V}=8.00 \mathrm{GeV}^{-2}$ [dashed line], $G_{V}=4.0 \mathrm{GeV}^{-2}$ [dotted line] and $G_{V}=0.0 \mathrm{GeV}^{-2}$ [dot-dashed line]. It would be of 


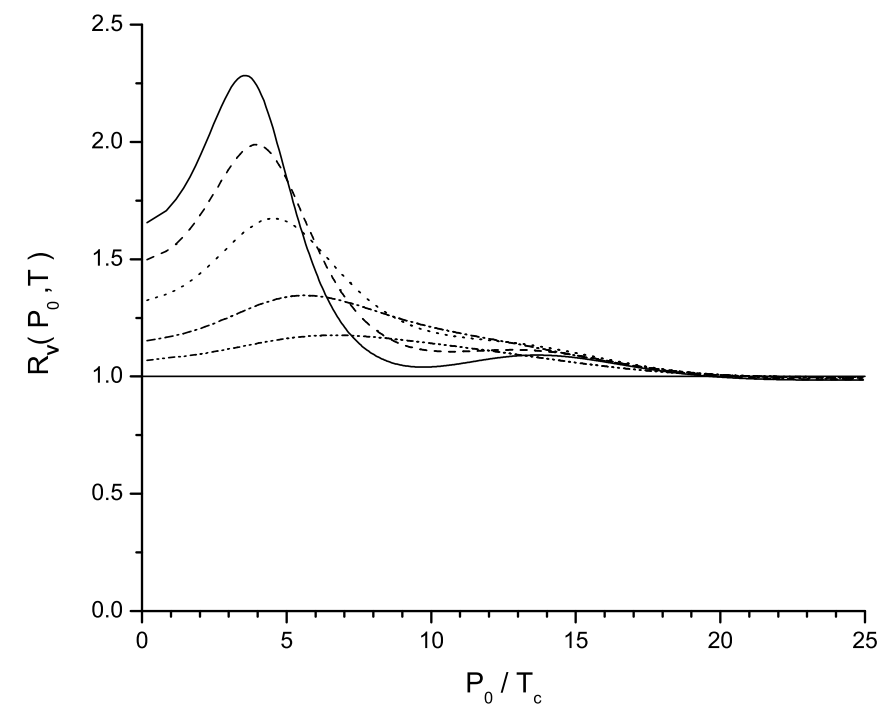

FIG. 14: Same caption as Fig.6, except that we show $R_{V}\left(P_{0}, T\right)=\operatorname{Im} C_{V}\left(P_{0}, T\right) / \operatorname{Im} C_{V}^{(0)}\left(P_{0}, T\right)$.

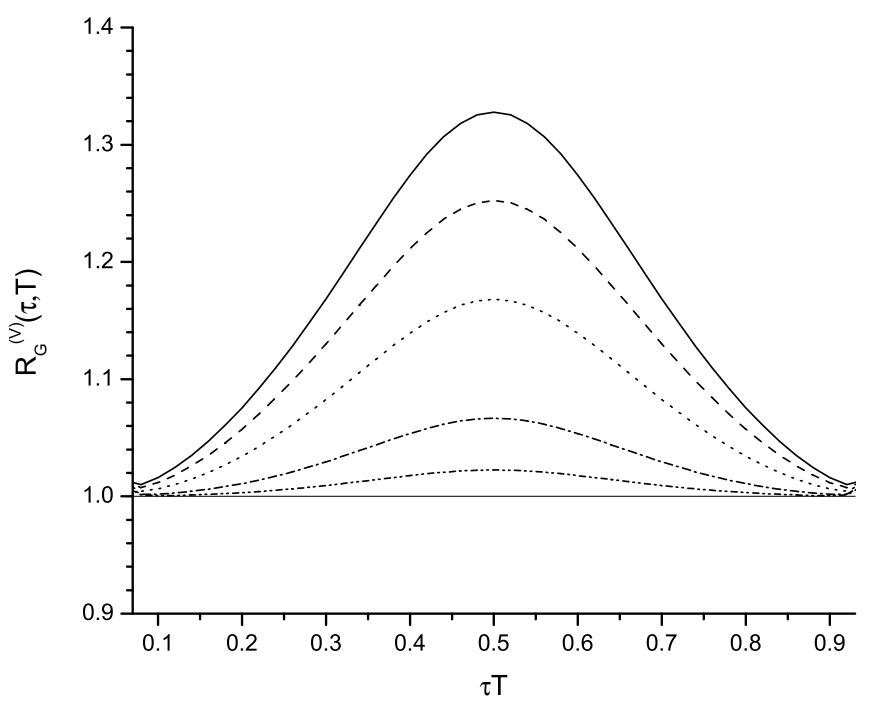

FIG. 15: Values of $R_{G}^{(V)}(\tau, T)$ are shown for values of $T / T_{c}=1.2$ [solid line], $T / T_{c}=1.5$ [dashed line], $T / T_{c}=2.0$ [dotted line], $T / T_{c}=3.0$ [dashed-dotted line], and $T / T_{c}=4.0$ [double dot-dashed line]. For $T / T_{c}=5.88, R_{G}^{(V)}(\tau, T)=1$.

interest to consider the results of Ref. [15] for the Euclidean-space correlator to be correct and to then determine $G_{V}(T)$ so that we fit the values of $G_{V}(\tau, T) / T^{3}$ obtained in the lattice calculations. We defer such a project to a future work. 


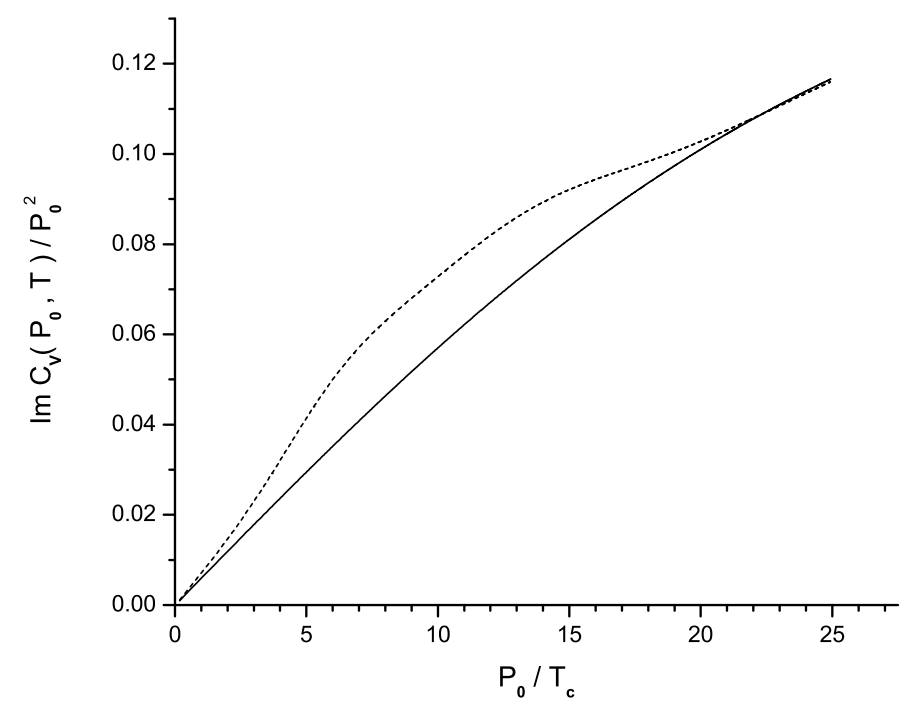

FIG. 16: Values of $\operatorname{Im} C_{V}\left(P_{0}, T\right) / P_{0}^{2}$ are shown for $T / T_{c}=5.88$. The dashed line represents the result when $G_{V}(T)=G_{V}=11.46 \mathrm{GeV}^{-2}$. The solid line is the result for $G_{V}(T)=0$, which is characteristic of models 1 and 2 , when $T / T_{c}=5.88$. The solid line, therefore, represents the values of $\operatorname{Im} \tilde{J}_{V}\left(P_{0}, T\right) / P_{0}^{2}$ for $T / T_{c}=5.88$.

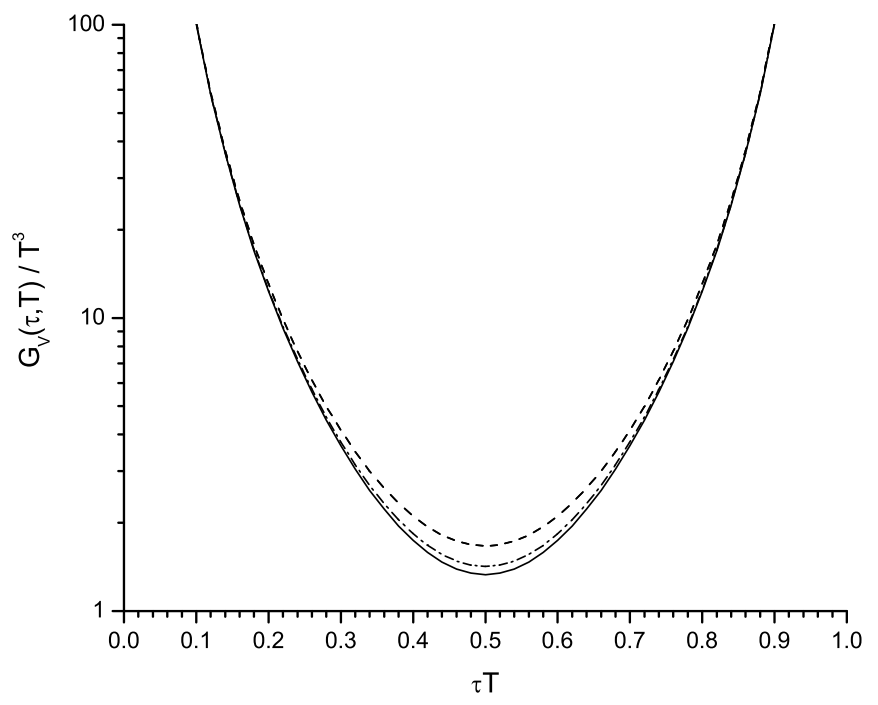

FIG. 17: Values of $G_{V}(\tau, T) / T^{3}$ are shown for $T / T_{c}=1.5$ [dashed line], $T / T_{c}=3.0$ [dashed-dotted line], and $T / T_{c}=5.88$ [solid line]. These results were calculated with model 1. 


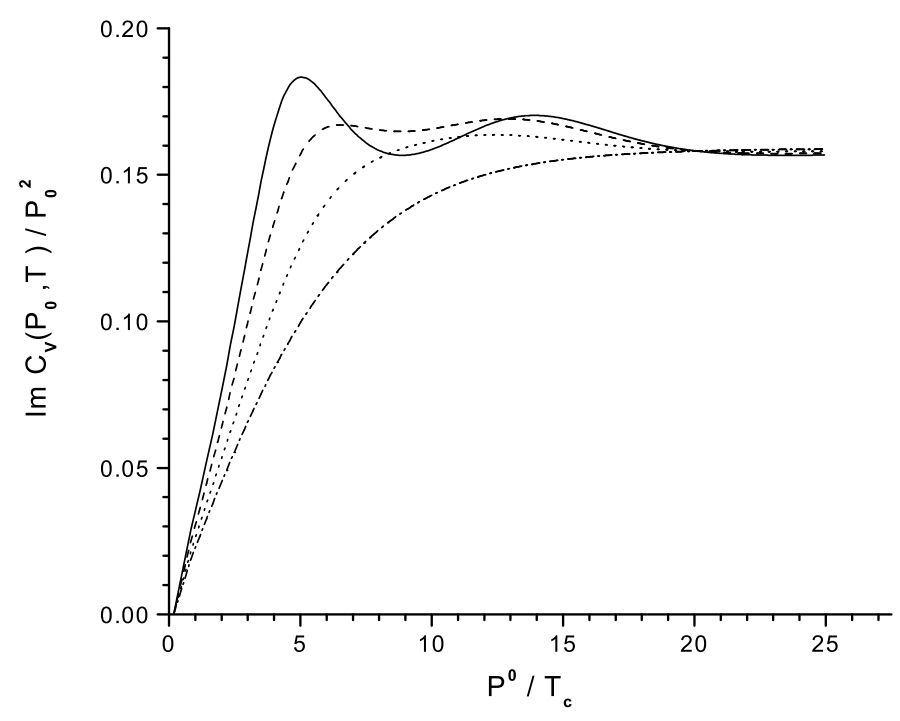

FIG. 18: Values of $\operatorname{Im} C_{V}\left(P_{0}, T\right) / P_{0}^{2}$ are shown for $T / T_{c}=1.5$ with $G_{V}=11.46 \mathrm{GeV}^{-2}$ [solid line], $G_{V}=8.00 \mathrm{GeV}^{-2}$ [dashed line], $G_{V}=4.00 \mathrm{GeV}^{-2}$ [dotted line] and $G_{V}=0.0 \mathrm{GeV}^{-2}$ [dot-dashed line]. Here, we have used model 1.

\section{DISCUSSION}

It is difficult to make a definitive comparison of our results and the results obtained for the spectral functions in the lattice simulations, since those results are accompanied by large errors. Also, it is somewhat difficult to understand why the different behavior seen for $G_{V}(\tau, T) / T^{3}$ and $G_{P}(\tau, T) / T^{3}$ in Ref. [15], leads to values for $\sigma_{P}(\omega, T) / \omega^{3}$ and $\sigma_{V}(\omega, T) / \omega^{3}$ that are rather similar [14-16]. On the other hand, the chiral Lagrangian model provides a systematic study, which may yield some guidance for further studies of lattice QCD. This may be particularly important in the light of the comments made in Ref. [15]:

"The reconstruction of $\sigma_{H}(\omega, T)$ and in particular the determination of its low energy structure thus is difficult at non-zero temperature. Additional complications arise in lattice calculations which necessarily are performed on lattices with finite number of points $\left(N_{\tau}\right)$ in Euclidean time. The correlation functions $G_{H}(\tau, T)$ can thus be calculated only at finite set of Euclidean times $\tau T=k / N_{\tau}$, with $k=0, \ldots N_{\tau}-1$. In order to reconstruct the spectral functions from this limited set of information it is necessary to include in the statistical analysis 
of numerical results also prior information on the structure of $G_{H}(\tau, T)$ as well as assumptions about the likelihood of a certain spectral function $\sigma_{H}(\omega, T)$. It has been suggested to provide this additional information through the application of the Maximum Entropy Method (MEM) [22, 23], which has been applied successfully to many other ill-conditioned problems in physics ..."

While it may be premature to compare our results for the spectral functions with those given in literature, the errors for the Euclidean-time correlation functions are small. Therefore, in Ref. [24] we have made an extensive comparison of our results with those presented in Ref. [15]. In that work we provided additional evidence for the temperature dependence of the coupling parameters that we have introduced.

[1] For reviews, see K. Rajagopal and F. Wilczek, in At the Frontier of Particle Physics/Handbook of QCD, M. Shifman ed. (World Scientific, Singapore 2001); M. Alford, Annu. Rev. Nucl. Part. Sci. 51, 131 (2001).

[2] Z. Fodor and S. D. Katz, hep-lat/0204029 Nucl. Phys. B (Proc. Suppl.) 106, 441 (2002) hep-lat/0106002; Phys. Lett. B 534, 87 (2002).

[3] Hu Li and C. M. Shakin, Phys. Rev. D 66, 074016 (2002).

[4] Bing He, Hu Li, C. M. Shakin, and Qing Sun, hep-ph/0211318.

[5] M. Le Bellac, Thermal Field Theory (Cambridge Univ. Press, Cambridge, 1996)-See Fig.1.3.

[6] Hu Li and C. M. Shakin, hep-ph/0209136.

[7] Hu Li and C. M. Shakin, hep-ph/0209258.

[8] C. M. Shakin and Huangsheng Wang, Phys. Rev. D 65, 094003 (2002).

[9] T. Hatsuda and T. Kunihiro, Phys. Rep. 247, 221 (1994).

[10] V. Vogl and W. Weise, Prog. Part. Nucl. Phys. 27, 195 (1991).

[11] L. S. Celenza, Bo Huang, Huangshen Wang, and C. M. Shakin, Phys. Rev. C 60, 025202 (1999).

[12] S. P. Klevansky, Rev. Mod. Phys. 64,649 (1992). [See Eq.(5.38) of this reference.]

[13] Bing He, Hu Li, C. M. Shakin, and Qing Sun, hep-ph/0203010. 
[14] I. Wetzorke, F. Karsch, E.Laermann, P. Petreczky, and S. Stickan, Nucl. Phys. Proc. Suppl. 106, 510 (2002).

[15] F. Karsch, S. Datta, E.Laermann, P. Petreczky, and S. Stickan, and I. Wetzorke, hep-ph/0209028.

[16] F. Karsch, E.Laermann, P. Petreczky, S. Stickan, and I. Wetzorke, Phys. Lett. B 530, 147 (2002).

[17] R. L. Kobes and G. W. Semenoff, Nucl. Phys. B 260, 714 (1985).

[18] C. M. Shakin, Wei-Dong Sun, and J. Szweda, Ann of Phys. (NY) 241, 37 (1995).

[19] F. Karsch, M. G. Mustafa, M. H. Thoma, Phys. Lett. B 497, 249 (2001).

[20] M. Asakawa, T. Hatsuda, and Y. Nakahara, hep-lat/0208059.

[21] T. Yamazaki et al., Phys. Rev. D 65, 014501 (2001).

[22] M. Asakawa, T. Hatsuda and Y. Nakahara, Prog. Part. Nucl. Phys. 46, 459 (2001)

[23] Y. Nakahara, M. Asakawa and T. Hatsuda, Phys. Rev. D 60091503 (1999).

[24] Bing He, Hu Li, C.M. Shakin, and Qing Sun, Brooklyn College Report No. BCCNT $03 / 021 / 319$. 